\title{
Monoclonal gammopathy and visceral leishmaniasis
}

\section{Gammapatía monoclonal y leishmaniasis visceral}

\author{
J. Ferreira ${ }^{1}$, ME. Centurión ${ }^{1}$, A. Armoa ${ }^{1}$, J. Zarza ${ }^{1}$
}

\section{Sr. Director:}

Las gammapatías monoclonales (GM) son un grupo de trastornos caracterizados por la proliferación clonal de células linfoides $\mathrm{B}$ en los últimos estadios madurativos que producen una inmunoglobulina (Ig) homogénea de carácter monoclonal denominada componente $\mathrm{M}^{1}$. Las GM se presentan principalmente en neoplasias hematológicas (discrasias de células plasmáticas) $\mathrm{y}$, con menor frecuencia, en enfermedades reumatológicas, autoinmunes e infecciosas. Ocasionalmente, puede encontrarse un componente $\mathrm{M}$ en individuos sin criterios diagnósticos de mieloma múltiple, macroglobulinemia de Waldeström, amiloidosis primaria u otros síndromes linfoproliferativos lo que recibe la denominación de GM de significado incierto ${ }^{2}$.

En muy raras ocasiones se ha reportado la presencia de una GM asociada a leishmaniasis visceral (LV) ${ }^{3-5}$.

Presentamos el caso de un varón de 58 años, procedente de un ambiente suburbano, que consultó por palidez de tres meses de evolución. No refería fiebre ni otra sintomatología asociada. En la exploración física destacaban una palidez cutáneo-mucosa y una esplenomegalia moderada que era palpable a $5 \mathrm{~cm}$ del reborde costal izquierdo.
En el hemograma se observó anemia normocítica (hemoglobina: $114 \mathrm{~g} / \mathrm{l}$, hematocrito: $0,348 \mathrm{l} / \mathrm{l}$ ), glóbulos blancos de 8,4 x $10^{9} /$ l (neutrófilos: $72 \%$ ) y plaquetas normales de $290 \times 10^{9} / 1$, con un recuento de reticulocitos de $2 \%$. En el frotis de sangre periférica llamaba la atención el fenómeno de Rouleaux. El test de Coombs directo fue positivo. Las proteínas séricas estaban elevadas, con un aumento de los niveles de globulinas (proteínas totales: $10 \mathrm{~g} / \mathrm{dl}$, albúmina: 3,05 g/dl, gammaglobulinas: 4,85 g/dl, ratio $\mathrm{A} / \mathrm{G}$ de 0,44$)$. Se realizó determinación de Ig, objetivándose una Ig G de $6.225 \mathrm{mg} \%$ (rango de referencia: 710-1520 mg \%), con el resto de las Ig en rango normal. La B2 microglobulina se encontraba aumentada: 5 $\mathrm{mg} / \mathrm{l}$ (valor de referencia inferior a 1,9 mg/l) y la LDH era normal. La proteinuria de Bence Jones fue positiva y el calcio sérico normal. Se realizó una serie ósea que descartó la presencia de lesiones osteolíticas.

Ante la sospecha clínica de una discrasia de células plasmáticas, se realizó un aspirado de médula ósea constatándose una celularidad global aumentada, un ligero aumento cuantitativo de la serie eritroide con maduración normal y la presencia de un $8,5 \%$ de células plasmáticas, sin otras alteraciones resaltantes. Se realizó además
1. Departamento de Hematología. Hospital de Clínicas. Universidad Nacional de Asunción (Paraguay)

Recepción: 9 de septiembre de 2012

Aceptación provisional: 30 de octubre de 2012

Aceptación definitiva: 6 de noviembre de 2012

\section{Correspondencia:}

Dr. José Zarza

Departamento de Hematología.

Universidad Nacional de Asunción (Paraguay)

Dr. Montero y Dr. Mazzei. Casilla de Correo 1012 e-mail: jjzarza@yahoo.es 
A.

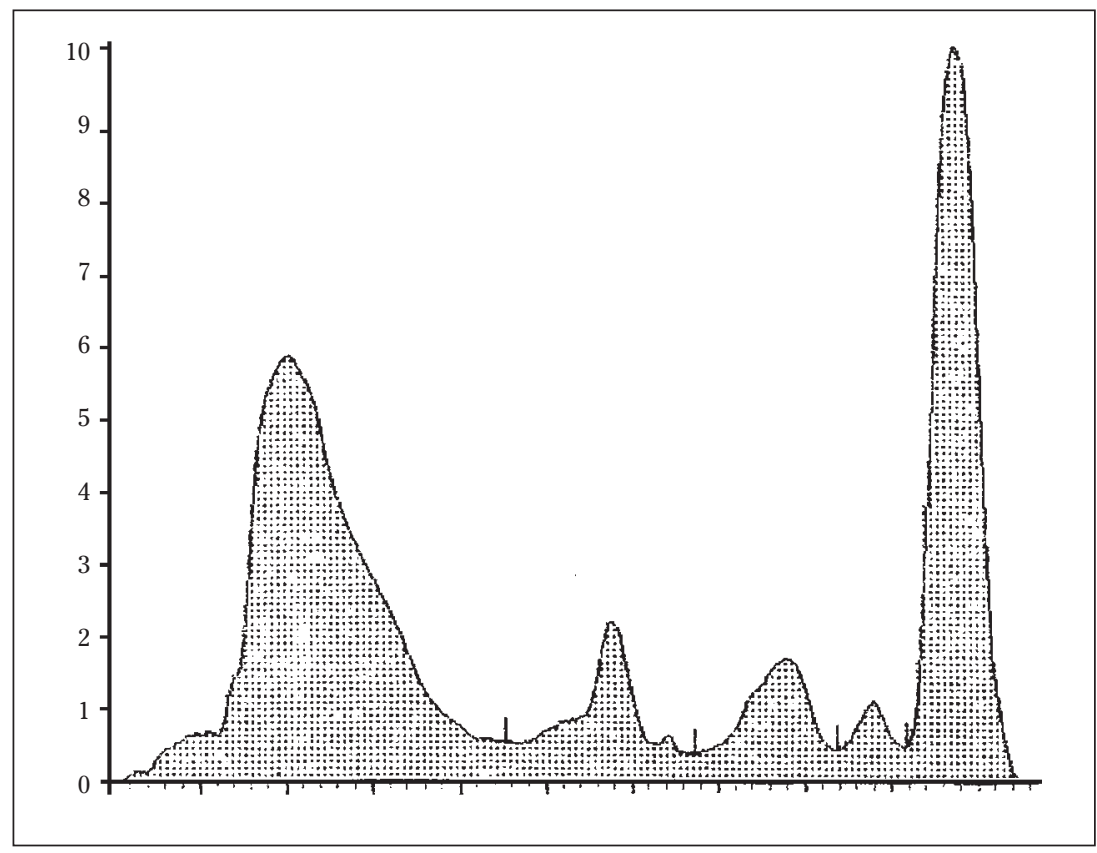

B.

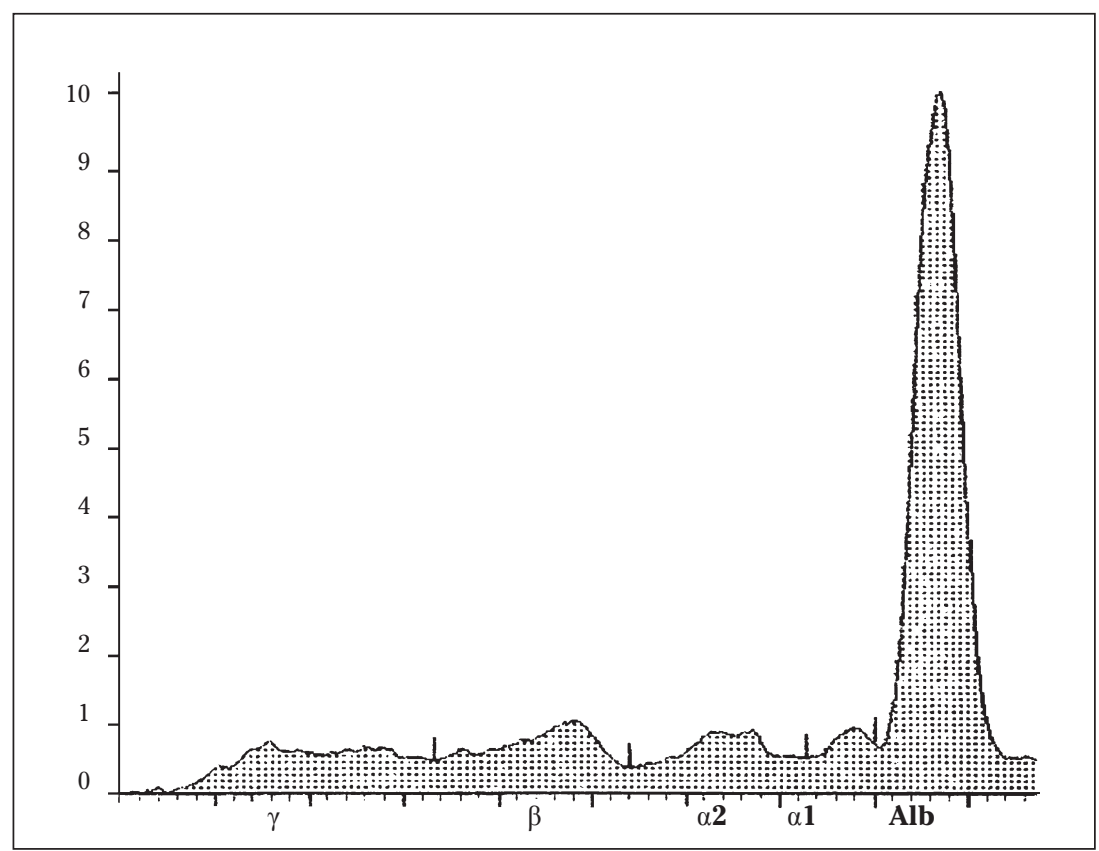

Figura 1. A. Proteinograma electroforético inicial, en el que se observa la presencia de componente M. B. Proteinograma electroforético posterior al tratamiento leishmanicida, en el que se constata la desaparición del componente M. 
una biopsia de médula ósea que informaba aumento de linfocitos y células plasmáticas de características reactivas que a la inmunohistoquímica eran positivas para cadenas ligeras kappa y lambda.

Con estos resultados, se interpretó como una anemia hemolítica autoinmunitaria y se inició tratamiento con prednisona, con la intención de realizar una nueva evaluación del paciente antes de decidir un tratamiento específico.

Veinte días después, acudió al servicio de Urgencias de nuestro hospital por una neumonía adquirida de la comunidad. Entre otros estudios, se solicitó un RK 39, que es una prueba serológica empleada para detectar anticuerpos específicos en contra de parásitos del género Leishmania pertenecientes al complejo Leishmania donova$n i$. Dicha prueba resultó positiva, indicando infección aguda. Un nuevo aspirado de médula ósea evidenció abundantes formas amastigotes de Leishmania donovani. En una posterior revisión del primer aspirado medular se observaron formas amastigotes del protozoo, pero en forma muy aislada, dentro y fuera de los macrófagos.

El paciente fue tratado con estilbogluconato de sodio durante un mes, con buena respuesta. La mejoría clínica se acompañó de desaparición de la anemia, la esplenomegalia y del componente $\mathrm{M}$ en el proteinograma electroforético.

En el caso presentado, el predominio de los signos de anemia y la ausencia de datos clínicos claros que orientaran hacia una LV, nos llevaron a pensar inicialmente en una discrasia de células plasmáticas como diagnóstico presuntivo. En el primer aspirado de médula ósea, las formas amastigotes de LV eran muy escasas y pasaron desapercibidas, pero luego del tratamiento con corticoides, se hicieron evidentes. Una enseñanza clara es que en casos similares, una LV debe ser siempre investigada, sobre todo en áreas endémicas como nuestro país.

En la literatura médica se ha descrito en ocasiones la presencia de una hipergammaglobulinemia policlonal asociada a LV, sin embargo, la detección de un componente M en estos pacientes es excepcional, existiendo sólo unos pocos casos publicados ${ }^{3-5}$. En
1998 García Menéndez y col, comunicaron el caso de 2 pacientes españoles procedentes de un área no endémica para LV, que tenían componente $\mathrm{M}$, en los que al igual que lo sucedido en nuestro paciente, existieron problemas para realizar el diagnóstico diferencial con otras entidades asociadas a la presencia de paraproteínas ${ }^{5}$.

Se debe diferenciar la GM asociada a LV de la GM de significado incierto, ya que esta última puede evolucionar a mieloma múltiple con el tiempo lo que condiciona un peor pronóstico ${ }^{2}$. Desde un punto de vista práctico, es conveniente destacar que cuando la GM está asociada a LV, el componente $\mathrm{M}$ desaparece con el tratamiento leishmanicida, lo que no sucede cuando se trata de una GM de significado incierto.

En nuestro paciente, no solamente desapareció el componente $\mathrm{M}$, sino que también lo hicieron la anemia y la esplenomegalia.

No está clara la etiología de las GM en pacientes con leishmaniasis, pero algunos autores sugieren que la fuerte estimulación antigénica determinada por la LV induce a su aparición en sujetos predispuestos ${ }^{6}$.

Finalmente, nos gustaría hacer hincapié en la necesidad de incluir la LV entre los diagnósticos diferenciales de las GM tanto en áreas endémicas como no endémicas.

\section{BIBLIOGRAFÍA}

1. García Sanz R, Mateos MV, San Miguel JF. Mieloma Múltiple. Med Clin (Barc) 2007; 129: 104-115.

2. BladÉ J. Monoclonal gammopathy of undermined significance. N Eng J Med 2006; 355: 2765-2777.

3. Sharma V, Agarwal MP, GiRi S. Monoclonal gammopathy associated with visceral leishmaniasis. Braz J Infect Dis 2010; 14: 297-298.

4. Randi ML, Ruzzon E, Tezza F, Tezza F, Pacquola E, FABRIS F. Monoclonal gammopathy in human leishmaniasis. Neth J Med 2006; 64: 50-51.

5. García Menéndez L, Santamaría López C, Fernández Eroles AL, Megido Lahera M, Galende del Canto J, Aguilera Sanz C. Monoclonal component in visceral leishmaniasis: a rare association that can lead to misdiagnosis. Rev Clin Esp 1998; 198: 517-520.

6. Rombola F, Spinoso A, Bertuccio SN. Monoclonal gammopathy after visceral leishmaniasis: just a coincidence? Infez Med 2008; 16 : 173-174. 